\title{
TUBERCULOSIS COMO ENFERMEDAD OCUPACIONAL
}

\author{
Alberto Mendoza-Ticona1,a
}

\begin{abstract}
RESUMEN
Existe evidencia suficiente para declarar a la tuberculosis como enfermedad ocupacional en diversos profesionales especialmente entre los trabajadores de salud. En el Perú están normados y reglamentados los derechos laborales inherentes a la tuberculosis como enfermedad ocupacional, como la cobertura por discapacidad temporal o permanente. Sin embargo, estos derechos aún no han sido suficientemente socializados. En este trabajo se presenta información sobre el riesgo de adquirir tuberculosis en el lugar de trabajo, se revisan las evidencias para declarar a la tuberculosis como enfermedad ocupacional en trabajadores de salud y se presenta la legislación peruana vigente al respecto.
\end{abstract}

Palabras clave: Tuberculosis; Enfermedades ocupacionales; Personas con discapacidad; Política de salud ocupacional; Salud laboral (fuente: DeCS BIREME).

\section{TUBERCULOSIS AS OCCUPATIONAL DISEASE}

\begin{abstract}
There is enough evidence to declare tuberculosis as an occupational disease among healthcare workers. In Peru, there are regulations granting employment rights regarding tuberculosis as an occupational disease, such as healthcare coverage for temporary or permanent disability. However, these rights have not been sufficiently socialized. This study presents information on the risk of acquiring tuberculosis in the workplace, and a review of the evidence to declare tuberculosis as an occupational disease among health care workers, presenting the current Peruvian law related.
\end{abstract}

Key words: Tuberculosis; Occupational diseases; Disabled persons; Occupational health policy; Occupational health (source: MeSH NLM).

\section{INTRODUCCIÓN}

Una de cada tres personas en el mundo está infectada por el bacillo de Koch, lo que convierte a la tuberculosis (TB) en la pandemia más importante a nivel mundial. Esto se debe a su eficiente mecanismo de transmisión por vía aérea y de persona a persona; a su capacidad de desarrollar enfermedad crónica, discapacitante y fatal, sobre todo en personas afectadas por el VIH/SIDA y, recientemente, a su capacidad de desarrollar resistencia a las drogas anti-TB disponibles. Todos estos factores de la enfermedad asociados con una débil respuesta del sistema de salud en países en desarrollo, han convertido a la TB en una de las más importantes amenazas a la salud pública a escala mundial (1). Es importante conocer que, del total de personas infectadas con el bacilo, no todas hacen la enfermedad activa pulmonar o extrapulmonar. Se estima que el $10 \%$ de los infectados desarrollarán la enfermedad activa a lo largo de su vida. La mitad lo hará en los primeros cinco años luego de infectarse con el bacilo.
En el Perú se estima que entre el 30 y $40 \%$ de la población está infectada por el bacilo de la TB, siendo mayor la proporción entre trabajadores de salud (por su contacto estrecho con personas con enfermedad activa sin adecuadas medidas de control de infecciones) (2) y en trabajadores del transporte público (por su contacto directo y cotidiano con diversas personas durante su jornada laboral) (3). Cada año en el Perú se notifican 32 mil casos nuevos de TB activa, lo que hace una tasa de morbilidad de 108 casos por 100 mil habitantes, cifra que coloca al Perú en el tercer lugar de los países con mayor carga de enfermedad tuberculosa en las Américas, precedido solamente por Haití y Bolivia (4). La TB afecta predominantemente a varones pertenecientes a la población económicamente activa (PEA). En la Figura 1 se muestra la distribución de los casos de TB según grupo etario en Perú, en el año 2011; destaca la mayor concentración de casos en la PEA nacional entre los 15 y 60 años, con un $80 \%$ del total de casos ${ }^{(4)}$.

\footnotetext{
Instituto de Medicina Tropical Alexander von Humboldt, Universidad Peruana Cayetano Heredia. Lima, Perú.

a Médico infectólogo tropicalista magister en Epidemiología Clínica
} 


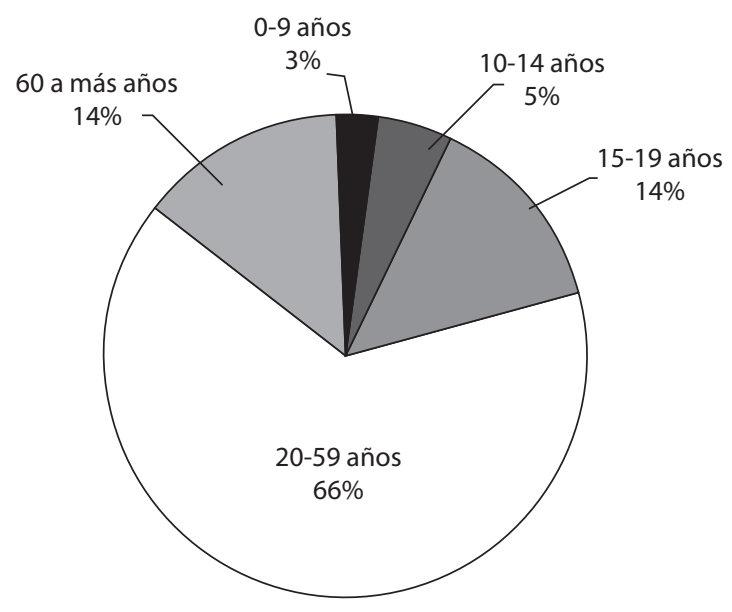

Figura 1. Distribución de casos de tuberculosis por grupos de edad, Perú 2011.

Fuente: Estrategia Sanitaria Nacional de Prevención y Control de la Tuberculosis del Ministerio de Salud, marzo 2012

La TB debe ser considerada una enfermedad ocupacional o profesional en aquellas personas que la contraen a causa de su trabajo (efecto de causalidad); es decir, que si no desempeñasen tal oficio o profesión, esta enfermedad no se hubiese producido o hubiese sido igual de frecuente que en la población general. En otras palabras, cuando la TB afecta al trabajador que está en contacto directo y frecuente con enfermos con TB, animales enfermos o materiales infectados con el bacilo tuberculoso, si no se demuestra otra forma de contagio no laboral, se la debe considerar una enfermedad profesional ${ }^{(5)}$.

En cambio, los casos de TB que se contraen en el lugar de trabajo debido a las relaciones interpersonales inherentes (efecto de casualidad), no se deben considerar como enfermedad profesional. Por ejemplo, adquirir la TB en el trabajo por contagio directo de un compañero con quien se comparte el mismo ambiente laboral hacinado, no es considerado un caso de TB ocupacional por que no es inherente al tipo de profesión u oficio del afectado sino a un problema de seguridad laboral.

En este artículo se hace una revisión del riesgo de contraer TB en el lugar de trabajo, la evidencia para declarar a la TB como enfermedad profesional en trabajadores de salud y la legislación nacional que regula la cobertura de seguros.

\section{RIESGO DE TUBERCULOSIS EN EL AMBIENTE LABORAL}

El mecanismo de transmisión de la TB es directo de persona a persona a través de la vía aérea. Esta forma de contagio es la más efectiva y la más difícil de prevenir.
Haciendo una comparación con el VIH, una persona puede prevenir eficientemente el contagio de este virus evitando relaciones sexuales por voluntad propia, pero no puede evitar respirar para prevenir contagiarse de TB de una persona con enfermedad activa que comparte estrecha y frecuentemente los mismos ambientes que pueden ser el dormitorio, el lugar de trabajo, el centro de estudio, el transporte público urbano, provincial o aéreo; lugares de reunión social, etc. Si consideramos que la mayoría de las personas trabajan en equipo entre los 16 a 65 años compartiendo el mismo espacio laboral ocho horas al día o cuarenta horas a la semana, es muy factible que, en estas circunstancias, la TB se pueda adquirir en el ambiente laboral por un efecto de casualidad ${ }^{(5)}$. Sin embargo, existen profesiones directamente asociadas con un mayor riesgo de padecer TB, como los mineros y la sílico-tuberculosis; los veterinarios y matarifes (zoonosis por M. bovis); trabajadores de funerarias; patólogos y personal involucrado en autopsias; personal de penitenciarías; casas de hospicio; transporte público; y personal de salud en general.

La probabilidad de infectarse depende del grado y tiempo de contacto con el bacilo. Por ejemplo, la sala de emergencia de hospitales; salas de broncoscopía o nebulización; servicios de neumología; salas de autopsia; laboratorios de cultivos de micobacterias; asilos; refugios; prisiones, etc. son lugares donde existe una mayor oportunidad de entrar en contacto con el bacilo; por lo tanto, las personas que laboran en estos ambientes están en riesgo de infectarse y padecer TB por consecuencia inherente a su ocupación, por lo que la seguridad laboral para el control de infecciones debe ser garantizada por el empleador.

La eficacia de la infección por $M$. tuberculosis depende de la concentración de partículas infectantes de Wells (de 1 a10 $\mu$ de diámetro); el medio transmisor (aire) y la susceptibilidad para infectarse del contacto ${ }^{(6)}$. Un acceso de tos puede eliminar 3000 partículas infectantes procedentes del pulmón o la laringe; en cambio, el esputo o la flema per se, no es un vehículo de transmisión, salvo que se deseque y se movilicen finas partículas con el aire. Otro factor importante es la distancia entre la fuente y el contacto. El riesgo de contagio depende de la distancia boca-boca entre la fuente y el contacto. A partir del medio metro de distancia la transmisión decrece logarítmicamente (a mayor distancia mayor número de metros cúbicos de aire, mayor dilución y menor cantidad de partículas infectantes) ${ }^{(5)}$. Por ello, son fundamentales para reducir la transmisión: los espacios amplios y ventilados con buen recambio de aire e iluminación natural, el uso de fuentes de luz UV artificial en la parte alta de las habitaciones y un grado bajo de humedad ambiental ${ }^{(6)}$. 
En cuanto a la susceptibilidad del contacto a infectarse, parece obedecer básicamente a los factores exógenos ya tratados, y al hecho de haber sido infectado previamente por el bacilo. En cambio, la susceptibilidad a enfermarse, es decir a desarrollar la enfermedad pulmonar o extrapulmonar activa, se debe a la integridad de la respuesta del sistema inmunitario, el cual puede afectarse por eventos exógenos o por marcadores genéticos. Por ejemplo, el polimorfismo del alelo CCL2-2518G incrementa el riesgo de desarrollar TB en personas de Asia y Latinoamérica (7). Dentro de los factores asociados a desarrollar enfermedad activa podemos mencionar a la infección por el $\mathrm{VIH}$; las infecciones recientes por $M$. tuberculosis; las lesiones fibróticas pulmonares sin antecedente de tratamiento; la silicosis; el tratamiento con antifactor de necrosis tumoral alfa; la terapia con inmunosupresores, y la insuficiencia y trasplante renal. Otros factores con menor evidencia son: la diabetes, el embarazo, el tabaquismo, la desnutrición proteica, las edades extremas de la vida, las enfermedades malignas del sistema linfático, entre otros ${ }^{(6)}$

\section{TUBERCULOSIS COMO ENFERMEDAD OCUPACIONAL EN EL PERSONAL DE SALUD}

La transmisión de la TB en los servicios de salud, tanto entre pacientes como entre personal de salud, ha sido descrita en casi todas las partes del mundo sin importar la incidencia local de TB. Desde la década de 1950 la TB empezó a ser considerada como una amenaza para el personal de salud, por lo que se empezó a implementar actividades específicas de aislamiento y protección respiratoria para reducir el riesgo de desarrollar TB nosocomial. Sin embargo, la insuficiente implementación de estas medidas en países en desarrollo, la epidemia del VIH y la emergencia de la TB resistente a diferentes fármacos, ha hecho reemerger esta forma de transmisión ${ }^{(8)}$. La experiencia de la epidemia de TB multidrogorresistente (TB-MDR) nosocomial que afectó a pacientes $\mathrm{y}$ al personal de salud en hospitales de New York a principios de los años noventa, contribuyó básicamente en dos aspectos: se logró la evidencia suficiente sobre la capacidad de transmisión activa de la TB-MDR, y la posibilidad de control a corto plazo cuando hay decisión política y capacidad técnica/financiera disponibles, algo que deberíamos seguir los países en vías de desarrollo ${ }^{(9)}$.

La mayor evidencia que confirma que la TB es una enfermedad ocupacional para el personal de salud viene de tres revisiones sistemáticas recientes. Los resultados del metanálisis de Baussano et al. ${ }^{(10)}$ muestran que el riesgo de TB, tanto en su forma latente como activa, entre personal de salud es consistentemente mayor que el riesgo entre la población general en todo el mundo. Los autores informan un riesgo anual de infección de TB entre trabajadores de salud de 4,6\% (IC 95\% 4,1 $5,6 \%)$ y una diferencia de riesgo de incidencia anual de TB de 2,9 (IC 95\% 2,4 - 5,1) entre trabajadores de salud frente a la población general. La ventaja de este estudio es que incluye investigaciones de diferentes países con niveles de carga de enfermedad bajos, intermedios y altos. Las otras dos revisiones sistemáticas publicadas por Menzies et al. y Joshi et al. ${ }^{(11,12)}$ también encuentran que la infección y la enfermedad por el bacilo tuberculoso, son eventos relacionados al trabajador de salud en todos los países, sobre todo en aquellos de bajos y medianos ingresos donde las medidas de control de infecciones son limitadas y la exposición es frecuente.

En Perú, la evidencia de infección tuberculosa del personal de salud se limita a informes de brotes en hospitales nacionales ${ }^{(2)}$. Escombe et al. ${ }^{(13)}$ describen en el personal de emergencia del Hospital Nacional Dos de Mayo de Lima una tasa anual de infección de $30 \%$; es decir, que treinta de cada cien personas que trabajan en estas salas de emergencia se infectan por el bacilo a lo largo de un año de seguimiento. Esta alta transmisión nosocomial de TB se asoció con un insuficiente número de medidas administrativas de control de infecciones: ausencia de triaje de casos sospechosos de TB; nula promoción de la etiqueta de tos; no usar mascarillas de papel en personas que tosen; ausencia de instalaciones de aislamiento de los casos detectados, y un limitado e inadecuado uso de respiradores con filtros N95. A pesar de haberse capacitado sobre el control de la transmisión de la TB en los principales hospitales de todo el país ${ }^{(14)}$, las cifras de casos de TB en personal de salud notificados por la Estrategia Sanitaria Nacional de Prevención y Control de la Tuberculosis del Ministerio de Salud, muestra una marcada tendencia a incrementar en los últimos años (Figura 2).

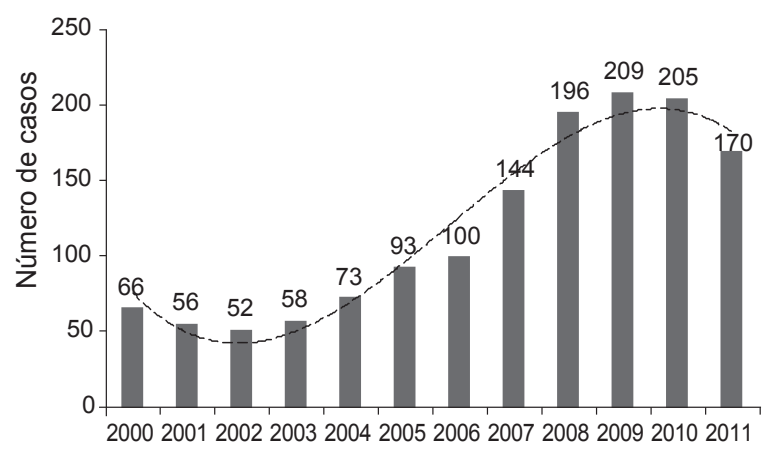

Figura 2. Casos de trabajadores de salud con tuberculosis, Perú 2000 - 2011.

Fuente: Estrategia Sanitaria Nacional de Prevención y Control de la Tuberculosis del Ministerio de Salud, marzo 2012. 


\section{LEGISLACIÓN NACIONAL}

En el Perú la TB es considerada como enfermedad profesional para el personal de salud en todo el territorio nacional, y está incluida en el Seguro Complementario de Trabajo Riesgo (SCTR), seguro obligatorio contemplado en la Ley de Modernización de la Seguridad Social (Ley 26790). El Ministerio de Salud, con RM 069-2011, aprobó el documento técnico: "Evaluación y calificación de la invalidez por accidentes de trabajo enfermedades profesionales" como reglamento de la ley que regula el SCRT. Dentro de la sección enfermedades infecciosas ocupacionales de este documento se desarrollan los criterios de evaluación y calificación de la invalidez temporal o permanente asociada con la TB ocupacional, sea pulmonar o extrapulmonar ${ }^{(15)}$.

Es importante que en nuestro país la TB haya sido declarada como una enfermedad profesional para el personal de salud; porque, además de brindar un derecho a una cobertura por invalidez temporal o permanente, debe determinar la puesta en marcha de medidas preventivas con el objeto de evitar su aparición en el resto de los trabajadores ${ }^{(16)}$.

En el país, una gran proporción del personal de salud tiene una modalidad de contrato temporal denominado Contrato Administrativo de Servicios (CAS). Según el Decreto Legislativo 1057 y su reglamento, las personas que prestan servicios bajo la modalidad de CAS también deben gozar de la cobertura adicional del SCTR cuando corresponda, para lo cual el empleador debe contratar este seguro a EsSalud, a la Oficina Nacional de Pensiones u otra compañía de seguros, siempre que desarrolle actividades de alto riesgo establecidas en el anexo 5 del Decreto Supremo 009-97-SA. El desafío actual consiste en hacer que los empleadores (principalmente el Estado) cumplan la ley y adquieran este tipo de seguro para el personal contratado por la modalidad CAS que atiende en los servicios de TB de todo el país.

Finalmente, el Ministerio de Trabajo y Promoción del Empleo, a través de su Dirección General de Derechos Fundamentales y Seguridad y Salud en el Trabajo, ha iniciado desde el 2010 actividades de promoción entre los empleadores y trabajadores para crear conciencia en la necesidad de espacios laborales libres de transmisión de TB y lucha frontal contra la discriminación de personas con TB en el centro de trabajo. Un afiche de esta campaña se muestra en la Figura 3. Actualmente, esta Dirección está elaborando un documento técnico que reglamente la obligatoriedad de ambientes con adecuada ventilación y medidas de prevención y vigilancia de la TB en el lugar de trabajo;

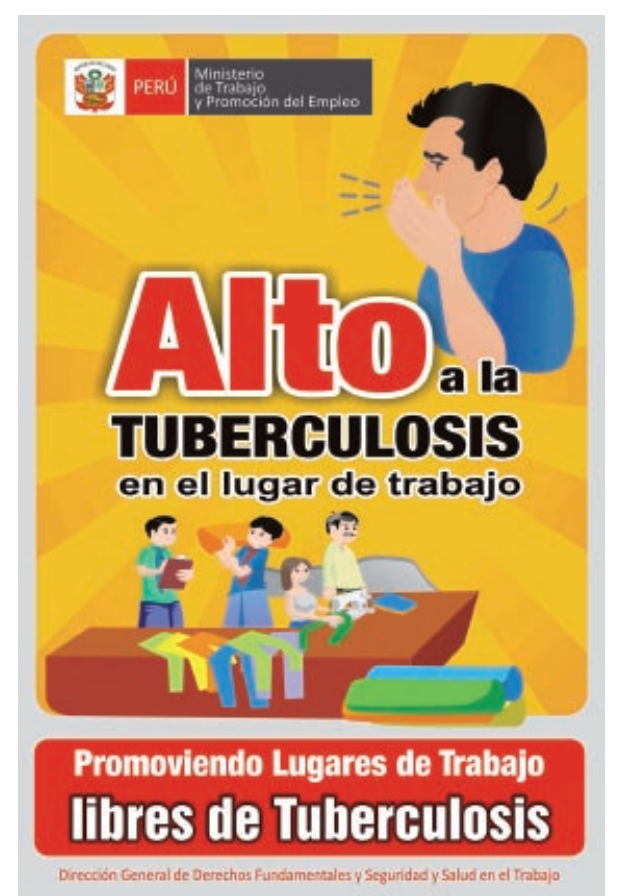

Figura 3. "Alto a la tuberculosis en el lugar de trabajo"; afiche promocional del Ministerio de Trabajo y Promoción del Empleo de Perú.

así como la disminución del estigma y la protección del empleo en los trabajadores afectados por la tuberculosis.

\section{AGRADECIMIENTOS}

Al Dr. Milton Palomino de la DG de Derechos Fundamentales y Seguridad y Salud en el Trabajo del Ministerio de Trabajo y Promoción del Empleo por su asistencia en temas legales. A la Lic. Claudia Salas por la revisión general del manuscrito.

A Perú ICOHRTA Network for AIDS/TB Research Training (NIH Grant 1U2RTW007368-01A1- Fogarty International Center, Dr. Gotuzzo, Lima Perú) por el entrenamiento recibido.

\section{Fuentes de financiamiento}

Autofinanciado.

\section{Conflictos de interés}

El autor declara no tener conflicto de interés.

\section{REFERENCIAS BIBLIOGRÁFICA}

1. World Health Organization (WHO). WHO Policy on TB Infection Control in Healthcare Facilities, Congregate Settings and Households. Geneva: World Health Organization; 2009.

2. Alonso-Echanove J, Granich RM, Laszlo A, Chu $\mathbf{G}$, Borja $\mathbf{N}$, Blas $\mathbf{R}$, et al. Occupational transmission of Mycobacterium tuberculosis to health care workers 
in a university hospital in Lima, Peru. Clin Infect Dis. 2001;33(5):589-96.

3. Horna-Campos OJ, Consiglio E, Sánchez-Pérez HJ, Navarro A, Caylà JA, Martín-Mateo M. Pulmonary tuberculosis infection among workers in the informal public transport sector in Lima, Peru. Occup Environ Med. 2011;68(2):163-5.

4. Perú, Ministerio de Salud. Situación de la tuberculosis en el Perú. Informe 2011. Lima: MINSA; 2011.

5. Rodríguez Bayarri MJ, Madrid San Martín F. Pulmonary tuberculosis as an occupational disease. Arch Bronconeumol. 2004;40(10):463-72.

6. Farga V, Caminero J. Tuberculosis. 3ra edición. Santiago de Chile: Mediterráneo; 2011.

7. Feng WX, Flores-Villanueva PO, Mokrousov I, Wu XR, Xiao J, Jiao WW, et al. CCL2-2518 (A/G) polymorphisms and tuberculosis susceptibility: a meta-analysis. Int J Tuberc Lung Dis. 2012;16(2):150-6.

8. Sepkowitz KA. Tuberculosis and the health care worker: a historical perspective. Ann Intern Med. 1994;120(1):71-9.

9. Beck-Sagué C, Dooley SW, Hutton MD, Otten J, Breeden A, Crawford JT, et al. Hospital outbreak of multidrugresistant Mycobacterium tuberculosis infections. Factors in transmission to staff and HIV-infected patients. JAMA. 1992;268(10):1280-6.

10. Baussano I, Nunn P, Williams B, Pivetta E, Bugiani M, Scano F. Tuberculosis among health care workers. Emerg Infect Dis. 2011;17(3):488-94.
11. Menzies D, Joshi R, Pai M. Risk of tuberculosis infection and disease associated with work in health care settings. Int J Tuberc Lung Dis. 2007;11(6):593-605.

12. Joshi R, Reingold AL, Menzies D, Pai M. Tuberculosis among health care workers in low- and middleincome countries: a systematic review. PLoS Med. 2006;3(12):e494.

13. Escombe AR, Huaroto L, Ticona E, Burgos M, Sanchez I, Carrasco L, et al. Tuberculosis transmission risk and infection control in a hospital emergency department in Lima, Peru. Int J Tuberc Lung Dis. 2010;14(9):1120-6.

14. Huaroto L, Espinoza M. Recomendaciones para el control de la transmisión de la tuberculosis en los hospitales. Rev Peru Med Exp Salud Publica. 2009;26(3):364-9

15. Perú, Ministerio de Salud. RM-069-2011. Evaluación y calificación de la invalidez por accidentes de trabajo y enfermedades profesionales [documento técnico]. Lima: MINSA; 2011.

16. Cascante JA, Hueto J. Tuberculosis como enfermedad ocupacional. An Sist Sanit Navar. 2005;28(Supl. 1):107-15.

Correspondencia: Alberto Mendoza Ticona

Dirección: Av. Cayetano Heredia 451-301. Lima 11, Perú.

Teléfono: (51) 980759549

Correo electrónico: mendozalberto@hotmail.com

\section{Consulte la versión electrónica de la Revista Peruana de Medicina Experimental y Salud Pública en}

\section{WWW.scopus.com}

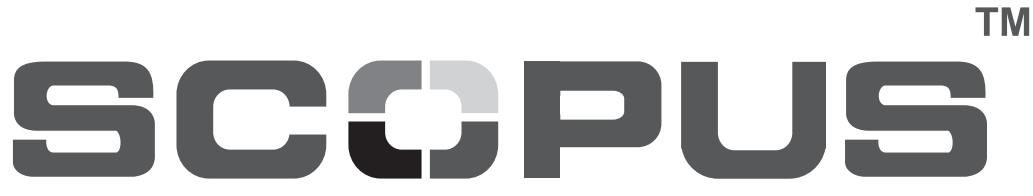

